\title{
Head and Neck
}

National Cancer Institute

\section{Source}

National Cancer Institute. Head and Neck. NCI Thesaurus. Code C12418.

For oncology, an area of the body generally construed to comprise the base of skull and facial bones, sinuses, orbits, salivary glands, oral cavity, oropharynx, larynx, thyroid, facial and neck musculature and lymph nodes draining these areas. 